\title{
Design Guidelines for the Development of Virtual Reality and Augmented Reality Training Systems for Maintenance and Assembly Tasks
}

\author{
Nirit Gavish* Teresa Gutierrez* ${ }^{\dagger} \quad$ Sabine Webel $\$ \quad$ Jorge Rodriguez* $* \quad$ Franco \\ Tecchia** \\ (*)Technion - Israel Institute of Technology, Israel \\ $(* \dagger) T E C N A L I A$, Spain \\ (†)Fraunhofer IGD, Germany \\ (*)CEIT - University of Navarra, Spain \\ (**)PERCRO - Scuola Superiore Sant'Anna, Italy
}

E-mail: niritg@tx.technion.ac.il,teresa.gutierrez@tecnalia.com, sabine.webel@igd.fraunhofer.de,jrodriguez@ceit.es,franco@sssup.it

\begin{abstract}
The current work describes design guidelines for the development of Virtual Reality (VR) and Augmented Reality (AR) platforms to train technicians on maintenance and assembly tasks of industrial machineries. The main skill involved in this kind of tasks is the procedural skill. Based on past literature and studies conducted within the SKILLS project, several main design guidelines were formulated. First, observational learning integrated properly within the training protocol increases training efficiency. Second, training protocols combining physical and cognitive fidelity enhances procedural skills acquisition. Third, guidance aids should be provided in a proper and controlled way. And last, enriched information about the task helps trainees to develop a useful mental model of the task. These recommendations were implemented in both VR and AR training platforms.
\end{abstract}

\section{Introduction}

The current work describes design guidelines for the development of Virtual Reality (VR) and Augmented Reality (AR) platforms to train technicians on maintenance and assembly tasks of industrial machineries. These machineries usually need complex and lengthy maintenance procedures to be performed periodically by teams of highly trained technicians. Industrial maintenance and assembly (IMA) training is fundamentally a combination of two elements: the comprehension of the basic working principles of the machines to be maintained, and, more important, the learning of complex procedures (sequence of steps) to be performed in order to assemble or replace some parts of the machine. Hence, the main skill involved in a maintenance and assembly task is the procedural skill. Procedural skills reflect the ability of the operator to obtain a good mental representation of how to perform each of the steps of a task and the correct order to perform them, which is reflected in their hierarchic organization [1]. They are based on both procedural memory and on a good and correct mental model of the machine, its components and required tools. Until now, coherent recommendations for designing VR and AR systems for procedural skills training in a general context, and for IMA activities training specifically, are still missing.

\section{Training platforms for industrial maintenance and assembly}

Within the SKILLS project ${ }^{1}$, two different platforms were developed for the training of IMA tasks, one based on Virtual Reality technologies (IMA-VR) and the other one based on Augmented Reality (IMA-AR). Using the IMA-VR system, the trainee learns the task within a virtual environment. In the IMA-AR system the trainee learns the task through assembly operations,

\footnotetext{
${ }^{1}$ SKILLS project (IST-2006-035005)
} 
which are linked to the real machines by superimposing virtual objects on the live camera image, and using the real instruments for interaction.

In the IMA-VR platform, the trainees interact with the virtual machine and tools in a multimodal way combining haptic, audio and visual feedback, see Figure 1. This platform provides different multimodal aids, not available in the real world, that help and guide the trainees during the training process. It also allows remote supervision/training through an on-line interaction between trainer and trainee.

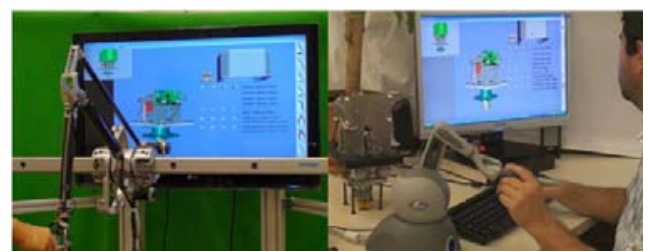

Figure 1. IMA-VR Training Platform: the trainee interacts and manipulates the virtual scene combining haptic, audio and visual feedback. The platform can be used with different types of haptic devices such as desktop workspace (right) and large workspace (left).

In the IMA-AR platform, the trainee interacts with the real machine and tools using a tablet PC that acts as interactive see-through device. For this purpose, a camera is attached to the tablet PC. A moveable mount allows for depositing the tablet PC and still using it as see-through device. Thus, the trainee has both hands free for physically performing the training task. Furthermore, the trainee is equipped with a vibrotactile bracelet that is used for providing additional information through haptic feedback. See Figure 2.

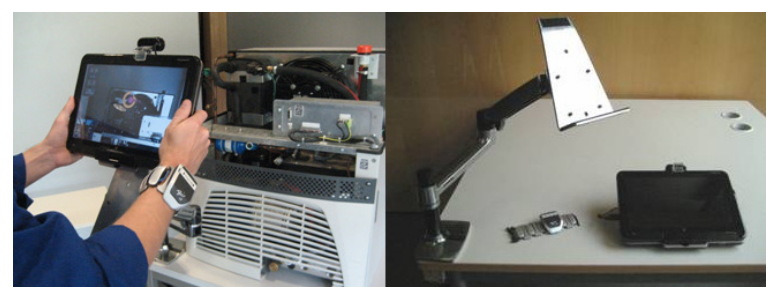

Figure 2. The trainee interacts with the IMA-AR platform using a tablet PC that acts as see-through device. Additional haptic feedback is presented to the trainee via a vibrotactile bracelet. The interactive training application is running on the tablet PC.

\section{Design guidelines: research and implication in the training platforms}

During the SKILLS project, several studies have been conducted in order to develop design guidelines for the VR and AR IMA training platforms. The fundamental research enabled us to provide applicative recommendations, which were applied in the platforms. Four main domains of research will be discussed: observational learning, cognitive fidelity versus physical fidelity, guidance aids and enriched information.

\subsection{Observational learning}

The common approach to training with VR training systems is the enactive approach, which stresses the importance of physical action within the environment to improve perception and enhance learning. However, under the theory of embodied cognition, verbal or visual stimuli are neurologically coupled with - and can prime - related motor activities. Hence, observational learning might replace active learning during some part of the training process. Two studies were conducted to address the use of observational learning in VR training systems.

The first study made use of a 75-steps Lego assembly task, where the trainees had to learn how to assemble a model helicopter [2]. Training was performed using a 3D haptic virtual reality system. Two conditions were compared: an active condition, in which trainees had to both identify each target brick and assemble it correctly in the Lego model; and a partly observational learning condition, in which trainees were required only to select the correct brick, which would then be automatically positioned by the system. Results showed that training time was reduced substantially with the incorporation of an observational learning phase, while final performance with the real Lego task was similar for both conditions. Thus, observational learning enhances training efficiency if it is integrated properly within Virtual Reality-based training. The second study [3] demonstrated that preliminary observational learning, in which the training starts with a demonstration, and the trainee performs the task only after acquiring a basic knowledge of the necessary steps, can shorten training time.

Based on these studies, preliminary observational learning strategy was implemented in the IMA-VR platform. In this strategy, instead of having an active interaction with the virtual scenario, trainees just watch the system which provides visual information about how to undertake the task in order to help trainees to develop a mental model of the assembly/disassembly process. This strategy is being used at the beginning of the training process.

\subsection{Cognitive fidelity versus physical fidelity}

Procedural skills are acquired through repeated exposure to a certain task. Hence, it is important to determine whether this task should be physically or 
cognitively represented within the simulator. The physical fidelity approach claims that the simulator should replicate the real-world task to the greatest degree possible. In contrast, the cognitive fidelity approach states that the simulator should engage the trainee in the type of cognitive activities involved in the real-world task, without needing to duplicate the physical elements of the task.

A study was conducted to compare real-world training and two alternative virtual trainers, one emphasizing the physical fidelity and the other the cognitive fidelity of the task [4]. Participants were randomly assigned to one of four training groups in the 75-steps Lego assembly task: Virtual-Physical Fidelity, Cognitive Fidelity, Real-World and Control. A posttraining test to assess the development of procedural skills was conducted. Results demonstrated the Cognitive Fidelity training was inferior in terms of test time compared with the Real-World training. In contrast, only the Real-World and the Cognitive Fidelity groups required significantly less time than the Control group for error correction. Conclusions are that the two training methods have complementary advantages, hence combining physical fidelity and cognitive training methods enhances procedural skills acquisition.

These outcomes were implemented in both platforms. In addition to the high physical fidelity training, the IMA-VR platform also provides cognitive training. The cognitive training is carried out by providing information about the current step not directly on the manipulated objects, but on a second display next to the virtual machine in which the system shows all the pieces and tools involved in the current step in their final position and a textual aid which describes the step and lists the names of the necessary pieces/tools (see Figure 3, left). This implementation allows cognitive training, since the trainees need to cognitively translate the display and the textual information to the actions that must be undertaken with the manipulated objects. Similarly, in the IMA-AR platform the information about the step and the required tools is presented near the real object instead of using traditional Augmented Reality overlays as 3D animations (see Figure 3, right).

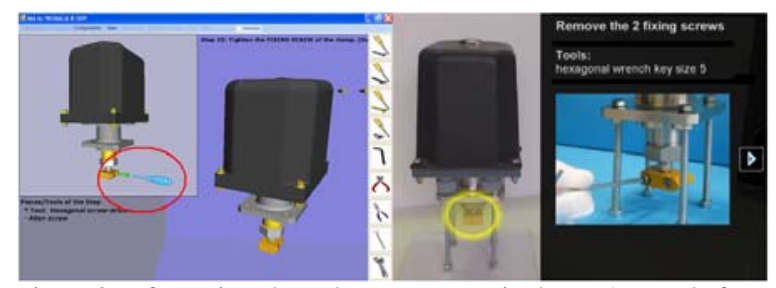

Figure 3. Information about the current step in the IMA-VR platform (left) and in the IMA-AR Platform (right).

\subsection{Guidance aids}

During the execution of a task, the trainees will sometimes need to receive direct information about how to proceed with the current step: guidance aids. However, the addition of guidance aids can have adverse effects on performance, because it encourages shallow performance strategies and little exploration. To examine this potential training trap, two experimental studies were conducted.

In the first study [5], each trainer instructed trainees on how to perform a $3 \mathrm{D}$ virtual puzzle in two conditions: Vocal guidance, where only vocal instructions were possible, and vocal guidance with mouse pointing, where the trainer could also use a mouse to point out the target positions on the trainee's screen. The results showed that while the use of the mouse pointer reduced trainees' mental load during training, it also drastically lowered performance level on a non-supervised test. These results suggest that the addition of guidance information should be considered very carefully. However, specific training protocols can improve the use of guidance tools.

This was the goal of the second study. This study made use of the 75-steps Lego assembly task and a 3D haptic virtual reality system. Two conditions were compared: building the virtual Lego model with the help of a printed instructions book (with step-by-step diagrams of the 75 stages); and building the virtual Lego with the help of direct aids provided by the virtual platform (change the color of the target brick and then show a copy in its the final position) but only on participants' demand. Both groups performed similarly in the real Lego test. Results showed that the use of guidance aids in a proper and controlled way, as providing aids only on trainees' demand, does not impede task learning.

Implementation of these outcomes in the platforms design is to provide guidance aids in a limited and controlled way, only when the trainee requests it. In the IMA-VR platform, the requested guidance aids are provided by means of visual aids (highlighting the target tool icon, changing the color of the target piece or displaying a copy of the target piece/tools in its final position) and haptic aids (exerting an attraction force towards the target piece/position). In the IMA-AR platform, although a superimposed $3 \mathrm{D}$-animation of a pulsing circle showing the part of the machine which is relevant for the performance of the current step is permanently presented, it provides only a hint about the required action, and the trainee needs to actively request additional information. 


\subsection{Enriched information}

Several past studies have demonstrated that when learners of a new procedural task are provided with enriched information about the task, in addition to the "how-to-do-it" knowledge, their performance becomes more accurate, faster and more flexible. This information enhances the building of the trainee's mental model about the task [6], and, as a result, improves performance. Taking into account that industrial maintenance tasks are based on complex procedures, it was proposed to divide the whole task into a set of logic sub-tasks. Providing enriched information about these sub-tasks could help trainees to develop an appropriate and more accurate mental model of the whole task.

A study was conducted to explore the efficiency of providing information in the step or sub-task level in an industrial task. In this study, the trainees used a haptic virtual environment to learn how to assemble part of an actuator. The task was composed of 23 steps grouped in five sub-tasks. Two conditions were compared: assembling the actuator receiving information in the step level or in the sub-task level. On the following day, participants had to assemble the real actuator. Results showed that although performance time was similar in both groups, the number of non-solved errors was larger in the group trained with step level information. The conclusions are that the use of enriched information should be encouraged in order to help the trainees develop a better mental model of the task.

Based on these outcomes both platforms provide the trainees enriched information by means of information in the sub-task level and information about the progress in the task. In the IMA-VR platform, enriched information in the sub-task level is provided in a second display in which the system shows the pieces involved in all the steps of the current sub-task in their final position (see Figure 4, left). Similarly, in the IMA-AR platform enriched information is provided with animated image of the current sub-task along with a progress bar (see Figure 4, right).

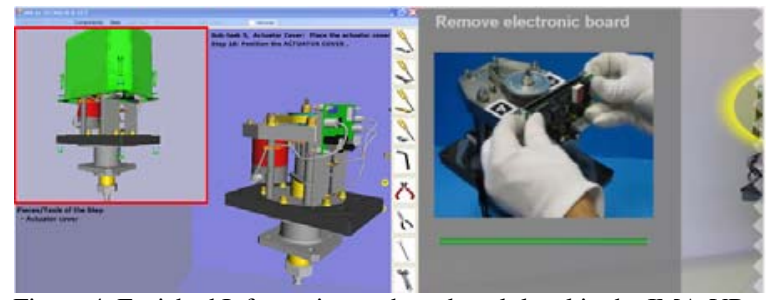

Figure 4. Enriched Information at the sub-task level in the IMA-VR platform (left) and in the IMA-AR Platform (right).

\section{Conclusions}

Based on past literature and studies conducted within the SKILLS project, several main design guidelines were formulated for the development of VR and AR training platforms in the IMA domain. The guidelines are: 1) observational learning integrated properly within the training protocol increases training efficiency; 2) training protocols combining physical and cognitive fidelity enhance procedural skills acquisition; 3) guidance aids should be provided in a proper and controlled way; and 4) enriched information about the task helps trainees to develop a useful mental model of the task. The recommendations were implemented in both IMA-VR and IMA-AR platforms and tested in a skills transfer evaluation performed by technicians.

\section{Acknowledgments}

The activities described in this paper were carried out with the financial assistance of the EU which cofunded the project SKILLS (IST FP6 ICT-IP-0350052006) the "Ministerio de Ciencia e Innovación" (Spain). Authors wishes to thank especially to all the scientists/technicians who helped in the development of the IMA training systems from DLR, FhG, CEIT, PERCRO, SIDEL, TECHNION and TECNALIA.

\section{References}

[1] J.R Anderson. Acquisition of cognitive skill. Psychological Review, 89, 369-406, 1982.

[2] N. Yuviler-Gavish, J. Rodríguez, T. Gutiérrez, E. Sánchez, and S. Casado. (Revised and re-submitted). Observational learning versus enactive approach in virtual reality training. Human Factors.

[3] N. Yuviler-Gavish, and M. Shelef (Submitted). Evaluating two modes of observational learning in cognitive-spatial task training. Applied Cognitive Psychology.

[4] I. Hochmitz, I., \& N. Yuviler-Gavish (Accepted). Physical fidelity versus cognitive fidelity training in procedural skills acquisition. Human Factors.

[5] N. Yuviler-Gavish, E. Yechiam, and A. Kallai. Learning in multimodal training: Visual guidance can be both appealing and disadvantageous in spatial tasks. International Journal of Human-Computer Studies, 69, 113-122, 2011.

[6] N.A. Taagten, D. Huss, D. Dickison, and J.R. Anserdon, The acquisition of robust and flexible cognitive skills. Journal of Experimental Psychology: General, 137, 548-565, 2008. 